\title{
Mechanisms of miniaturization of sessile drops on a heated surface
}

\author{
SHIGEAKI INADA $\dagger$ and WEN-JEI YANG \\ Department of Mechanical Engineering and Applied Mechanics, University of Michigan, \\ Ann Arbor, MI 48109-2125, U.S.A.
}

(Received 16 April 1991 and in final form 13 July 1992)

\begin{abstract}
The miniaturization phenomena of single water drops are experimentally studied following their impingement on a heated surface. Measurements are made on the frequency and amplitude of the elasticlongitudinal waves produced by boiling, and the acoustic pressure of boiling sounds using both a piezoelectric potential transformer and a condenser microphone. The process of morphological change and microexplosion is recorded by means of high-speed photography. It is disclosed that the maximum values of the frequency and amplitude of the elastic-longitudinal waves and the maximum values of the boiling acoustic pressure occur in a certain range of the heated surface temperatures for violent miniaturization of sessile drops. The range of the heated surface temperature coincides with that of transition boiling regime.
\end{abstract}

\section{INTRODUCTION}

A VAPOR explosion or explosive boiling may result, when a very hot liquid such as molten metal or salt is rapidly mixed with a cold, vaporizable liquid such as water. A similar phenomenon occurs when cold drops are placed on a hot surface. It is called micro explosion if the entire drop explodes on contact with a heated surface or is referred to as miniaturization if minute drops are expelled from the drop surface following the contact. The vapor explosion of molten metals and salts is of great concern to the safety of nuclear reactors, liquefied gas plants, molten metal processing, pulp industry and heat treatment. The micro explosion of fuel drops is of great interest in an enhancement of liquid fuel combustion.

There are two kinds of vapor explosions, depending upon the speed of heat transfer between the two media : large- and small-scale vapor explosions. The former involves two liquids at a large temperature difference such that the speed of heat transfer is comparable to or less than the acoustic relief time of the liquid-liquid system. A small-scale vapor explosion refers to the case in which heat is stored within the cold liquid on a time scale larger than the acoustic relief time.

Two schools of thought prevail on the major cause in triggering of a small-scale vapor explosion and in the triggering and propagation of larger-scale explosions, which result from coarse premixing of two liquids : spontaneous homogeneous nucleation (in the cold liquid) and film boiling destabilization. Henry and Fauske [1] proposed a spontaneous homogeneous nucleation model to describe the inception and propagation mechanisms for vapor explosions: The neces-

† Visiting scholar on leave from the Mechanical Engineering Department, Gunma University, Kiryu, Japan. sary conditions for an explosive boiling include (i) the threshold temperature for spontaneous nucleation in the cold liquid, (ii) the existence of stable film boiling to sustain vapor embryos of the critical size prior to vapor explosion, (iii) the size of cold liquid drops exceeding a critical value to be captured by the hotliquid surface, and (iv) the interfacial temperature between the two liquids exceeding the homogeneous nucleation value. Inoue and Bankoff [2] and Inoue $e t$ al. [3] proposed the destabilization of film boiling (vapor film collapse) as the mechanics of triggering and propagation of vapor explosion. Destabilization is favored by a decrease in the hot or cold liquid temperature (or both), an increase in relative velocity of the two fluids, or by a sudden increase in pressure. Ochiai and Bankoff [4] proposed the 'splash' theory, which states that 'splash' (instantaneous boiling or vapor explosion) occurs within the temperature range $T_{\mathrm{sn}}<T_{\mathrm{i}}<T_{\mathrm{cr}}$. Here, $T_{\mathrm{sn}}$ and $T_{\mathrm{cr}}$ denote the spontaneous nucleation and critical temperatures of the cold liquid, respectively, while $T_{\mathrm{i}}$ is the interfacial temperature between the two liquids. Iida et al. [5] conducted experiments on vapor explosions resulting from mixing a molten salt drop such as $\mathrm{LiCl}$ or $\mathrm{LiNO}_{3}$ in water. It was discovered that : (i) stable film boiling and $T_{i}>T_{\mathrm{sn}}$ are necessary conditions for vapor explosion in the molten salt-liquid system, and (ii) both the hot and cold liquids have the upper and lower temperature limits for vapor explosions. All the studies mentioned above suggest that film boiling is a necessary condition for vapor explosions which take place only within a certain temperature range depending on the combinations of the hot (molten salts and metals or liquid) and cold liquids. It is not yet conclusive which of the two theories, namly the destabilization of film boiling and the spontaneous nucleation-growth phenomena, describe the inception and propagation mechanisms for explosions.

Zhang and Yang [6] experimentally disclosed that a 


\section{NOMENCLATURE}

d drop diameter [mm]

E amplitude of the piezoelectric potential $[\mathrm{mV}]$

$E_{\text {tri: }}$ maximum peak-to-peak amplitude of piczoclectric potential $[\mathrm{mV}$ ]

$f$ frequency of piezoelectric potential [kHz]

$h$ falling height of drop [mm]

$\bar{q}_{\mathrm{w}} \quad$ time-averaged surface heat flux [W m ${ }^{2}$ ]

$T_{\mathrm{s}} \quad$ saturated water temperature [ $\left.\mathrm{C}\right]$

$T_{w} \quad$ heating surface temperature [ $\left.\mathrm{C}\right]$

$\bar{T}_{w} \quad$ time-averaged heating surface temperature $[\mathrm{C}$ ]
$T_{\mathrm{wi}} \quad$ initial temperature of heated surface [ C]

$\Delta T_{\text {suh }}$ subcooling $[\mathrm{K}]$

$u_{\mathrm{i}} \quad$ drop impacting velocity, $(2 g h)^{1}$ ? [mm s ']

We impinging drop Weber number, $\rho_{\mathrm{r}} u_{\mathrm{i}}^{2} d / \sigma$.

Greek symbols

$\beta \quad$ maximum acoustic pressure [Pa], [dB]

$\rho_{\mathrm{r}} \quad$ liquid density $\left[\mathrm{kg} \mathrm{m}^{-3}\right]$

$\sigma \quad$ surface tension $\left[\mathrm{N} \mathrm{m}^{-1}\right]$

$\tau \quad$ time after drop impacts [ms]

$\tau_{\mathrm{m}} \quad$ time at which $T_{\mathrm{w}}$ takes a minimum value [ms]. drop underwent a micro explosion on a heated surface whose temperature is in the transition-boiling type evaporation regime, suggesting the spontaneous nucleation model for the mechanism of micro explosion. Inada ct al. [7] performed transient surface temperature measurements following the impingement of discrete water drops on a heated surface in the transition boiling (rebounding) regime, with the liquid at 2-84 $\mathrm{C}$ subcooling. It is disclosed that: (i) the liquid-solid interfacial temperature falls to a minimum at about one half of the drop residence time followed a rapid recovery to the initial surface temperature, and (ii) the boiling curve in a sessile drop takes an $\mathrm{M}$-shape, characterized by two maxima with a saddle point in between, instead of an $\mathrm{N}$-shape as in the pool boiling case. The study has confirmed that the miniaturization of sessile drops occurs in the transition boiling regime.

Experimental evidence indicates that the miniaturization of sessile drops on a heated surface is related to the liquid-solid interfacial phenomena and the formation of vapor films. Inada $e t$ al. [8] used a photoelectric transformer to investigate the vapor film behavior in drops during miniaturization. In a subsequent study [9], water drops (of two different diameters 2.3 and $4.0 \mathrm{~mm}$ ) at $88^{\circ} \mathrm{C}$ subcooling were placed on a heated surface with the impinging Weber numbers ranging from 12 to 98 . The initial temperature of the heated surface, $T_{\mathrm{wi}}$, was varied from 160 to $420 \mathrm{C}$. Measurements were made on the heated surface temperature $T_{w}$ immediately following the drop impingement. The miniaturization process was photographically recorded and analyzed by means of image processing on the photographs. It was revealed that: (i) the miniaturization phenomena occurs only within a certain range of $T_{w}$, named as miniaturization boiling regime; (ii) $T_{\mathrm{w}}$ remains constant or increases during the formation of vapor films. During the miniaturization process, $T_{\mathrm{w}}$ falls abruptly. This implies the collapse of vapor films and the occurrence of instantaneous vaporization (like in nucleate boiling) resulting from a direct contact between the liquid and solid phases subsequent to the vapor film collapse; (iii) In addition to the miniaturization boiling regime, threc more regimes are disclosed depending upon the magnitude of $T_{\text {wi }}$ : nucleate boiling. latent-heat transport, and thin vapor-film boiling regimes.

Boiling heat transfer performance in a single water drop impinging on a heated surface was investigated by Inada et al. [7, 10, 11]. Measurements were made on the timewise variations of both the liquid-solid interfacial temperature and heat flux following the drop impact. The initial heating-surface temperature (prior to drop impact) $T_{\mathrm{wi}}$ was varied in the range from 160 to $420 \mathrm{C}$. Takano and Kobayashi [12-14] experimentally studied the vaporization of a single water drop impinging on a heated surface with flamesprayed ceramic coating, such as aluminum, magnesium zirconate and zirconium oxide. Results were obtained for the drop lifetime versus the heating surface temperature and the droplet-surface contact area versus the heat flux.

Impacting spray heat transfer can be divided into two categories: individual droplet impacting heat transfer and spray impacting heat transfer (for example refs. $[15,16])$. Choi and Yao [15] investigated the macroscopic mechanisms of film boiling heat transfer of a liquid spray impacting on a hot surface. The contribution of each heat transfer mechanism (droplet-wall impacting heat transfer, air convective heat transfer and thermal radiative heat transfer) to the overall impacting heat transfer. The study reported that the horizontal spray provided better heat transfer than a corresponding vertical case in the transition boiling regime, most likely due to easier vapor removal from the surface. No explanation was provided on the microscopic heat transfer mechanism in the transition-boiling regime.

The previous studies have confirmed that the micro explosion and miniaturization of sessile drops occur in the transition boiling regime, resulting from an instantaneous vaporization induced by vapor film collapse. However, the mechanics of miniaturization has been left unexplored. The present study is to determine 
the parameters which trigger the miniaturization process of liquid drops. The following methods are used in achicving the objective: (i) the miniaturization process and the behavior of minute vapor bubbles inside the drop are photographically recorded by mcans of a high-speed camera ; (ii) a condenser microphone is used to detect the boiling sound generated from the heated surface: (iii) the elastic-longitudinal waves produced by boiling are monitored using a piezoelectric potential transformer.

\section{EXPERIMENTAL APPARATUS AND PROCEDURE}

Figure 1 is a schematic diagram of the experimental apparatus used in the present study. (1) was the drop generating nozzle through which the drops of degassed, distilled water fell intermittently. In testing, the shutter (2) was moved to allow only one drop of 4 $\mathrm{mm}$ in diametcr to fall $40 \mathrm{~mm}$ onto the central portion of the heated surface (3). By washing the nozzle surface with constant temperature water, the drop from the nozzle was maintained at $15 \mathrm{C}$. The heated surface was the upper end of a $15 \mathrm{~mm}$ in diameter, $100 \mathrm{~mm}$ long, copper cylinder, its upper end was plated with a $0.03 \mathrm{~mm}$ thick platinum by means of diffusion welding in order to prevent an oxidization of the heated surface. The heated surface was finished with an emery paper $5 / 0$. The cylinder was heated by the Nichrome- wired electrical furnace (5) via the carbon cylinder (4). The cylinder temperature was adjusted by means of the slide trunk (1).

The heated surface temperature was monitored by means of the thermocouples (7) and recorded on the recorder $\$$. Copper-constantan thermocouples of 50 $\mathrm{mm}$ diameter were spot-welded on the cylindrical side surface near the upper end. Argon gas was blown into the test section from the lower end of the electrical furnace to prevent the oxidization of the cylinder. diffusion-welding part and thermocouples.

The lower end of the healing cylinder was flangeshaped, sitting on a pair of R-T cut quartz (D) with 20 $\mathrm{mm}$ diameter and $10 \mathrm{~mm}$ thickness. A tight contact between the flange and the quartz was achieved by means of a pair of copper plates (iI and bolts, as shown in Fig. 1. Each surface of the quartz was gold-plated (by vapor deposition method) and connected in parallel for recording piezoelectric potentials.

Upon contact with the heated surface, boiling sounds are generated at the liquid-solid interface. Elastic-longitudinal $(E)$ waves which are induced by boiling sounds propagate through the heating surface into the quartz in less than $0.05 \mathrm{~ms}$. The boiling sounds were detected by means of a condenser microphone (1) (with resonance frequency of $30 \mathrm{kHz}$ ), while the $E$ waves were monitored using a piezoelectric potential transformer (quartz oscillator). Two signals

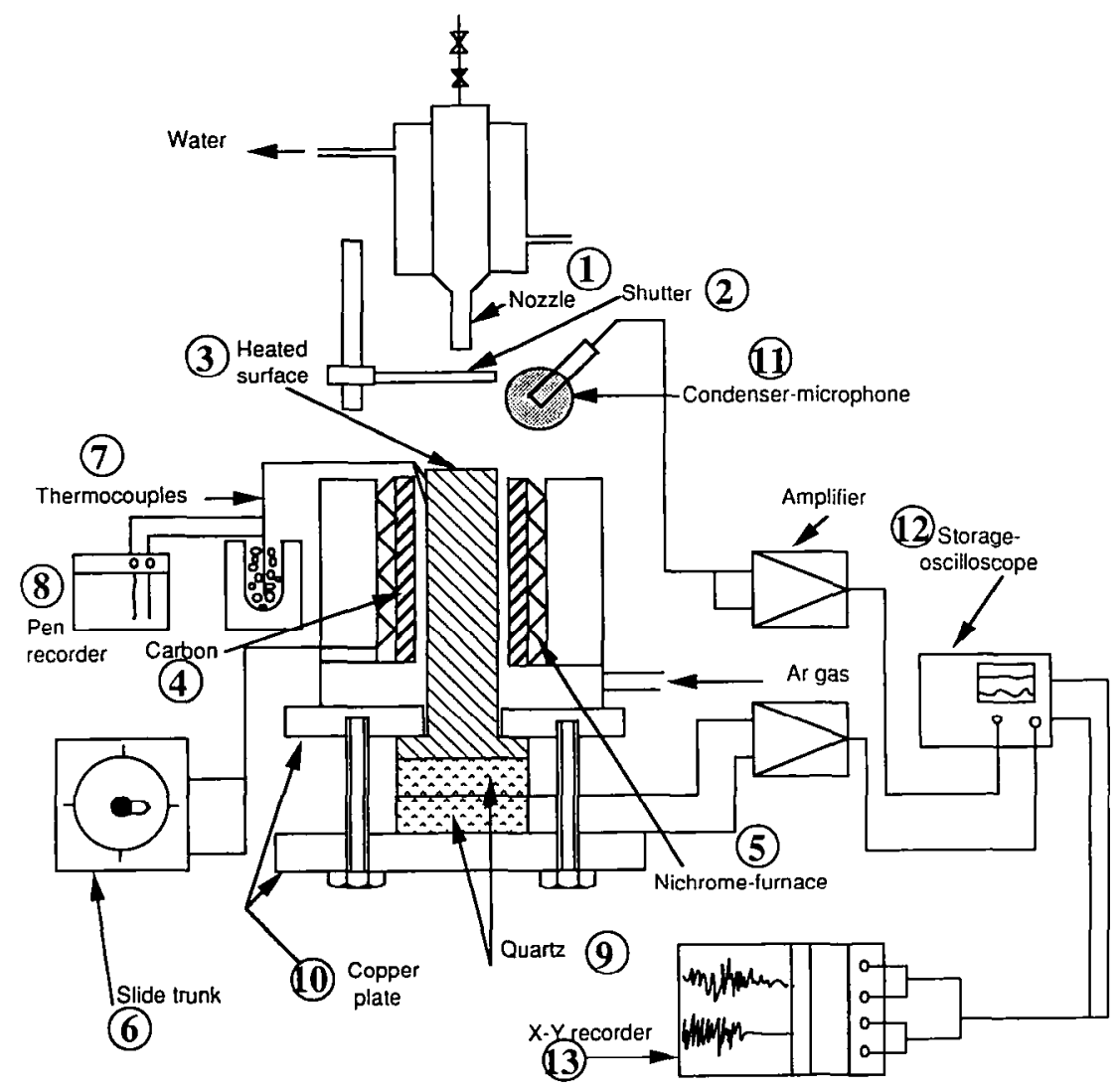

FIG. 1. A schematic diagram of experimental apparatus. 
were memorized in the storage oscilloscope (1) (with frequency characteristics of $60 \mathrm{MHz}$ ) through the amplifiers (with frequency characteristics of $30 \mathrm{kHz}$ and input impedance of $100 \mathrm{M} \Omega$ ) and simultaneously recorded by the $X-Y$ recorder (13).

\section{RESULTS AND DISCUSSION}

\section{Miniaturization process}

Figure 2 illustrates a series of photographs taken on a drop immediately following its impingement on the platinum heated surface at different initial tcmperature $T_{w i}$. The time after impact $\tau$ is indicated below each photograph. A splash in white color indicates a miniaturization process in progress. The values of $T_{\mathrm{wi}}$ and $\tau$ corresponding to the occurrence of miniaturization process are listed in Table 1 . White spots inside the drops are the suspension of micro vapor bubbles that were generated on and detached from the heated surface. These bubbles filled up the entire drops within $3 \mathrm{~ms}$ after impingement when $T_{\text {wi }}$ was in the range between 200 and $340^{\circ} \mathrm{C}$.

Two conditions are required for the occurrence of miniaturization: (i) after impingement, the drop spreads over the heated surface and becomes a disk shape, and (ii) the heated surface temperature is in the transition boiling regime. Under these circumstances, an examination of the photographs reveal that numerous minute vapor bubbles are formed inside the drop on the heated surface immediately following the liquid-solid contact. Each bubble grows very rapidly, detaches from the heated surface with a sufficiently high energy to shoot upward through the drop, and blasts through the drop surface carrying a multiple of minute drops into the atmosphere.

Two important phenomena are observed in Fig. 2. (1) After a violent miniaturization process is over, the drop resembles a circular disk marked with huge craters. The drop on a surface at $T_{\mathrm{wi}}=280^{\circ} \mathrm{C}$ and $\tau=12.0 \mathrm{~ms}$ in Fig. 2 is an example. Thereafter, surface tension force reshapes the spread and disfigured drop into a spherical form, which rebounds from the heated surface (not shown). (2) In addition to the transition boiling regime, the miniaturization process takes place at a lower temperature range, $T_{w i}=160-180^{\circ} \mathrm{C}$. The main difference in the miniaturization behaviour between the higher and lower temperature ranges is

Table 1. Initial heating-surface temperature and time (after drop impingement) for initiation of miniaturization process of $4.0 \mathrm{~mm}$ diameter water drops on platinum surface at $\Delta T_{\text {sub }}=85 \mathrm{~K}$ and $W e=43$

\begin{tabular}{cc}
\hline$T_{\mathrm{wi}}\left[{ }^{\circ} \mathrm{C}\right]$ & $\tau[\mathrm{ms}]$ \\
\hline 160 & After 12.0 \\
180 & $15.0-20.5$ \\
260 & $3.0-6.0$ \\
280 & $2.5-8.5$ \\
300 & $3.5-8.5$ \\
320 & $6.0-8.0$ \\
\hline
\end{tabular}

that no elastic longitudinal wave vibration occurs preceding the miniaturization process in the lower temperature case, as will be seen later in Fig. 3. In the lower temperature case, the drop is spread thin (Fig. 2) and the nucleation, growth and departure of vapor bubbles are at a slower pace and have less energy because of a lower $T_{\text {wi }}$. Consequently, no elastic wave vibration is triggered.

\section{Elastic longitudinal waves (E waves)}

Figure 3 shows elastic longitudinal waves monitored by the piezoelectric potential transformer, at various values of $T_{\mathrm{wi}} \cdot \tau=0$ corresponds to the instant of drop impingement on the platinum heating surface. The occurrence of miniaturization process is indicated by circles whose size shows the relative strength of miniaturization. It is observed that a violent miniaturization process takes place after large oscillation of $E$ waves. In the case of $T_{\mathrm{wi}}=160^{\circ} \mathrm{C}$, the miniaturization occurs after the drop has spread into a thin liquid layer. It implies that drop shape is also a factor in miniaturization. The magnitudes of both the amplitude and frequency of $E$ waves determine whether the drop will go into the spheroidal state or miniaturization process.

Figure 4 depicts the temperature history of the platinum heated surface for a $4 \mathrm{~mm}$ diameter drop. The height of drop impingement was changed to vary the Weber number. $T_{w}$ denotes the timewise surface temperature of the heating surface. $T_{w}$ fell rapidly immediately after drop impingement $(\tau=0)$, and rebound after reaching a minimum value at the time $\tau_{m}$. The magnitude of $\tau_{\mathrm{m}}$ diminishes with Weber number (We) but increases with $T_{\text {wi. }}$. The variation of the surface temperature history is summarized as follows :

(i) $T_{w i}=160-180^{\circ} \mathrm{C}:$ The rebounding of $T_{w}$ is weak and remains in the range of $130-140^{\circ} \mathrm{C}$ for a long time, suggesting a good wetting of the heated surface. A weak miniaturization was observed during this time, with a dispersion of minute liquid clusters from the drop surface. The drop is in the state of subcooled 'nucleate boiling'.

(ii) $T_{\mathrm{wi}}=200-240^{\circ} \mathrm{C}$ : After rebounding of $T_{\mathrm{w}}, T_{\mathrm{w}}$ remains in the range of $140-150^{\circ} \mathrm{C}$ for a while during which latent-heat transport prevails in the drop. The drop is in the state of 'latent-heat transport'. It is characterized by the appearance of tiny bubbles throughout the entire drop from the moment of impingement till $\tau=2.5 \mathrm{~ms}$.

(iii) $T_{\mathrm{wi}}=260-340^{\circ} \mathrm{C}:$ In the range of $T_{\mathrm{w}}=180$ $200^{\circ} \mathrm{C}$ corresponding to the transition boiling regime, a violent dispersion of minute liquid clusters is observed. These clusters are much finer than those seen in the range of $T_{w i}=160-180^{\circ} \mathrm{C}$. The miniaturization phenomenon is induced by an instant vaporization (explosive boiling) resulting from a direct contact between part of the drop and the heating surface upon the destruction of the vapor film which is formed after drop impingement. The drop is in the state of 


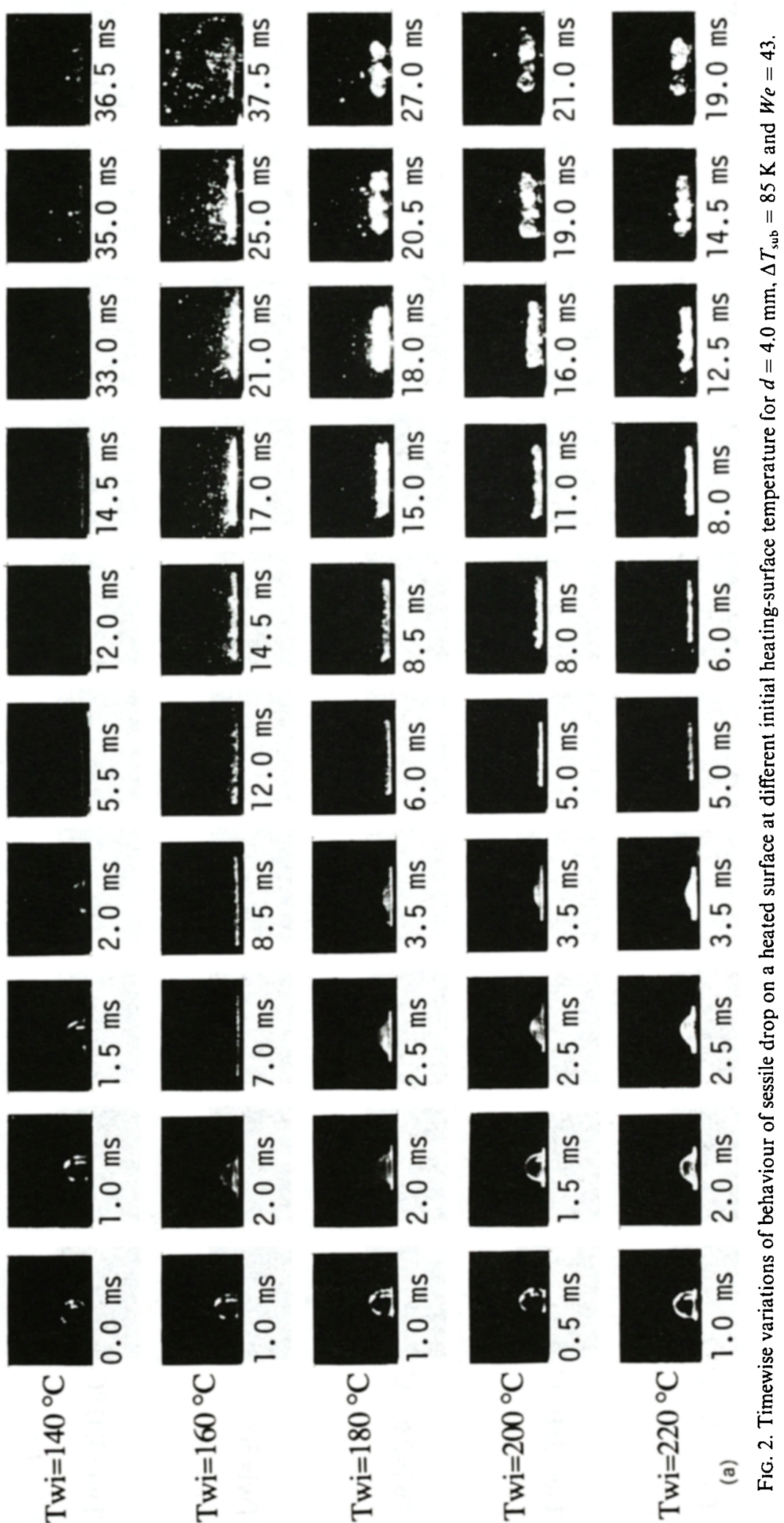




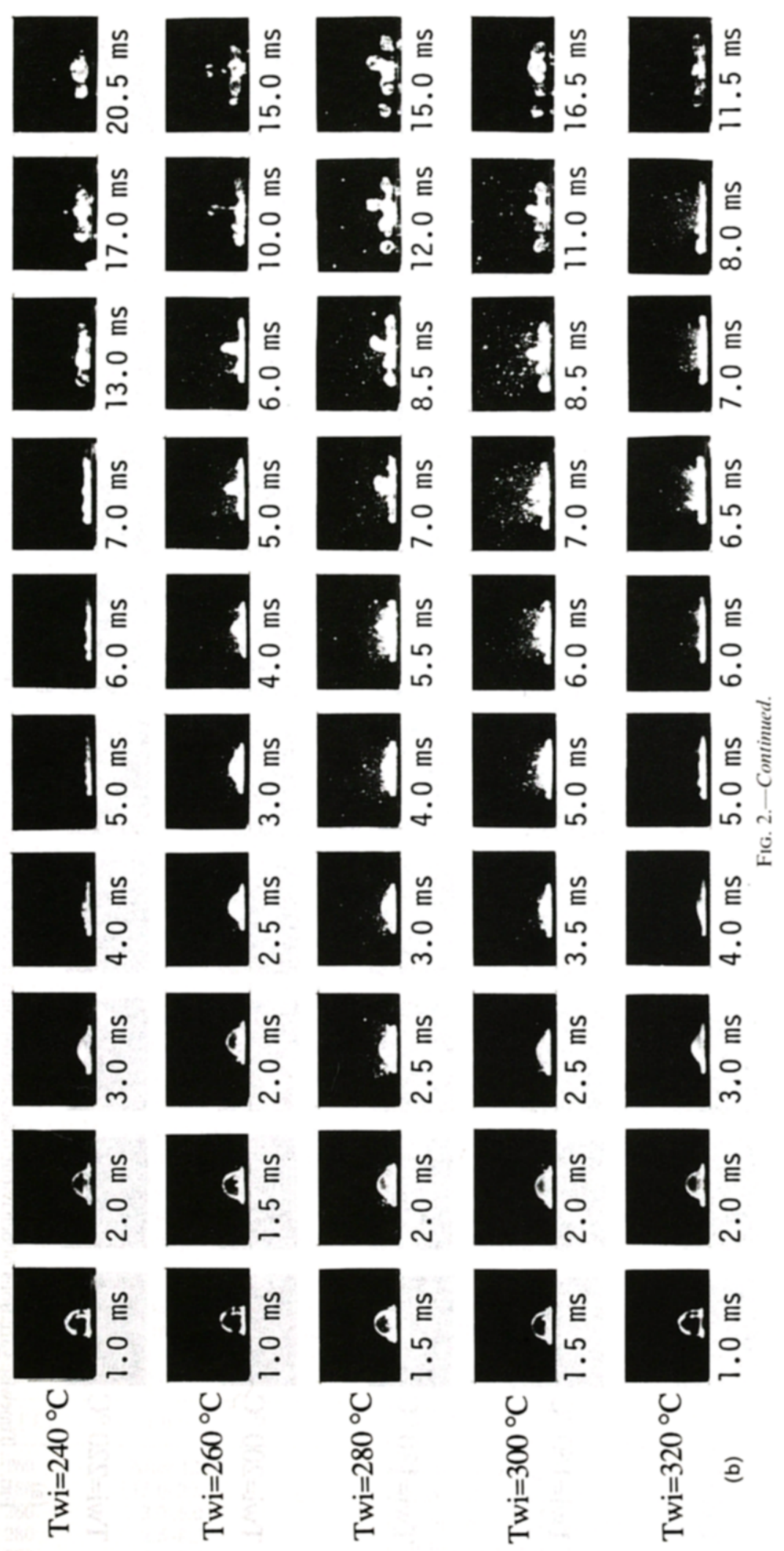




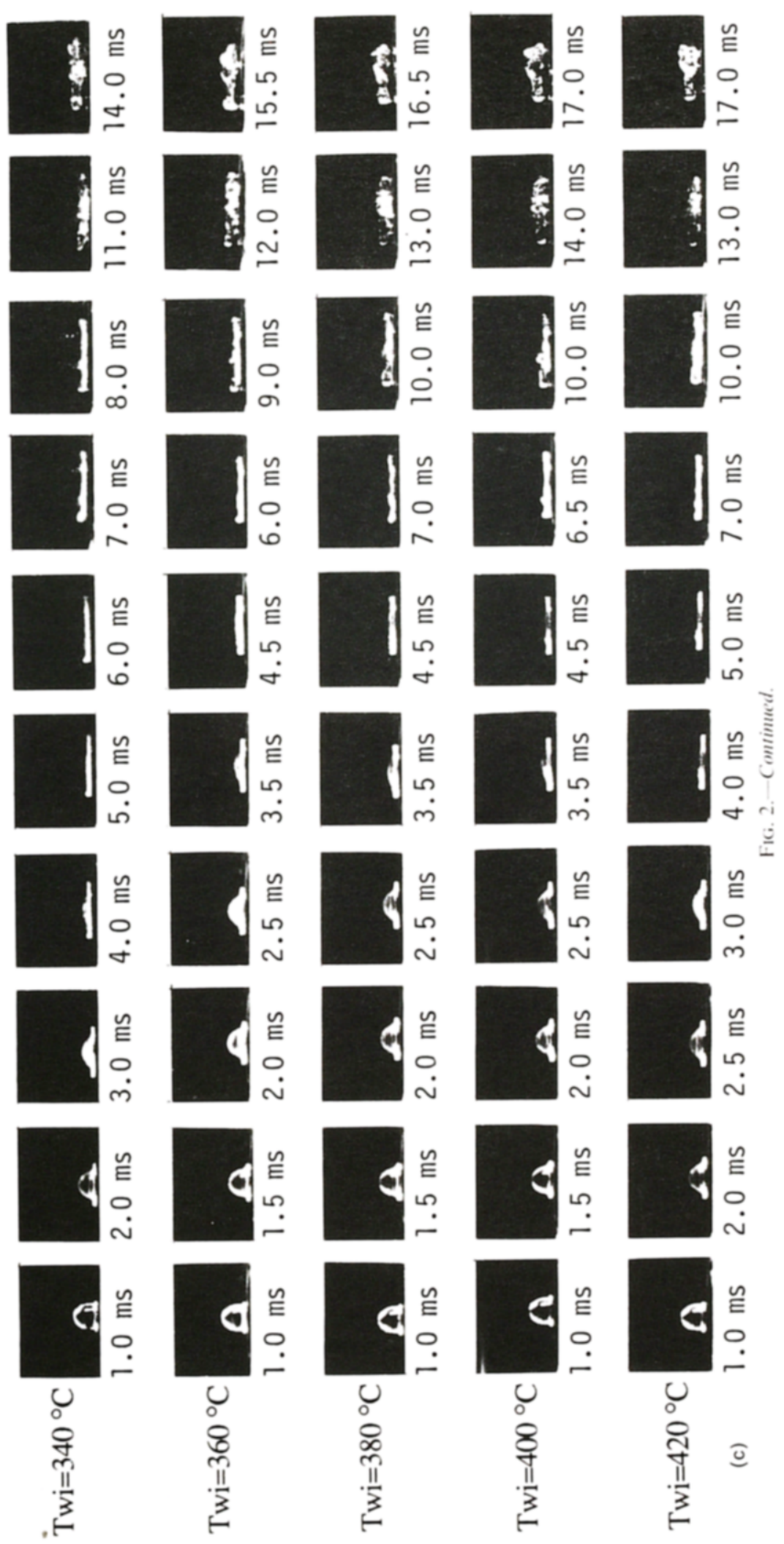




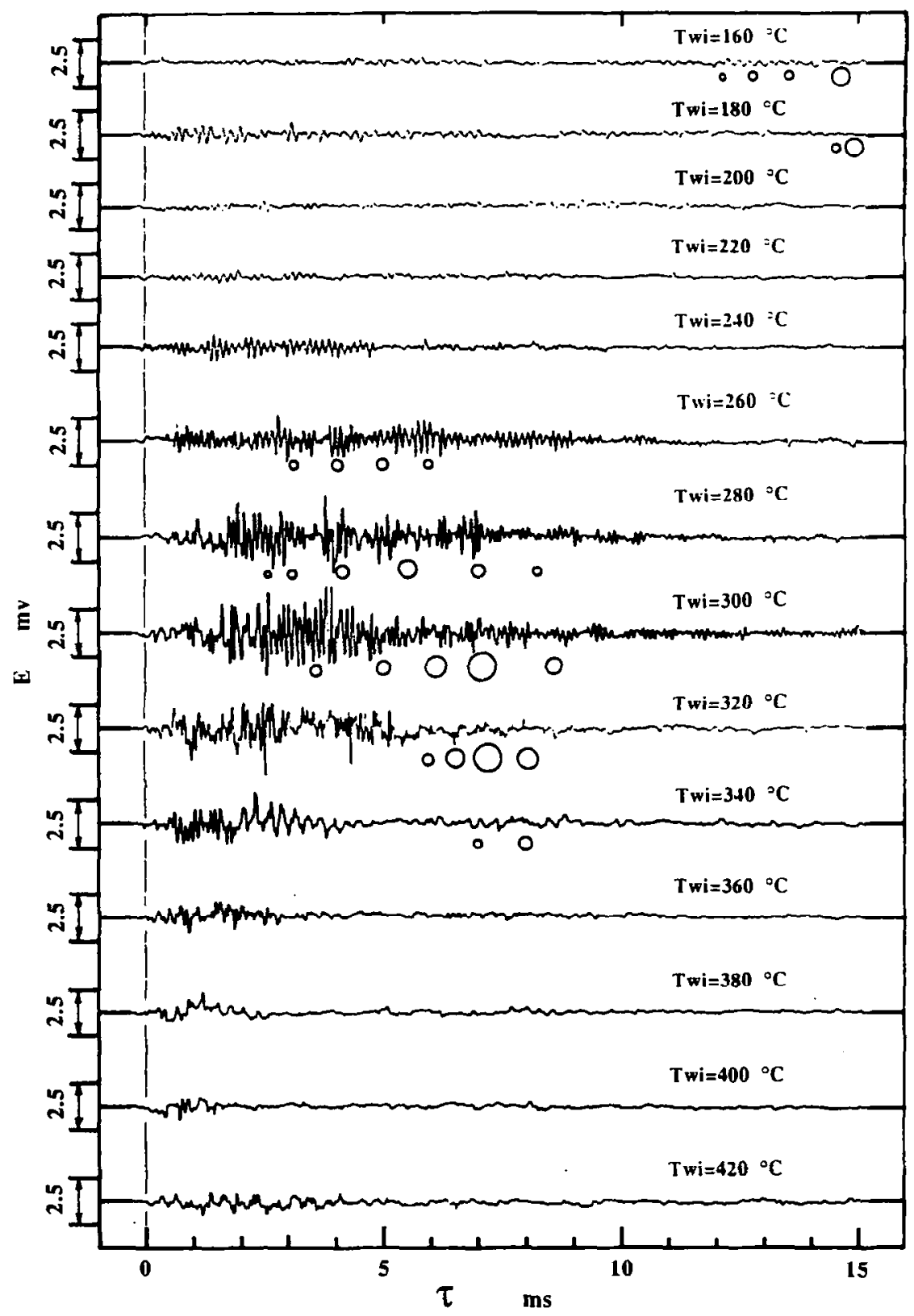

FIG. 3. Recordings of piezoelectric potential and minute liquid clusters (in circles representing different cluster size) for various initial heating-surface temperatures.

'miniaturization boiling'. The range of $T_{\mathrm{w}}$ for the occurrence of miniaturization phenomena is identified by slashes.

(iv) $T_{\mathrm{wi}}=360-420^{\circ} \mathrm{C}$ : The surface temperature curves exhibit an inflection point (especially in low Weber number cases) in the course of temperature fall immediately following drop impingement. It is evidence of the formation of a stable vapor film between the drop and the heated surface, as experimentally observed. The drop is in the state of 'film boiling'.

\section{Acoustic pressure and piezoelectric potentials}

Figure 5 plots the virtual value of the maximum acoustic pressure $\beta$ in decibels, and the frequency $f$ in $\mathrm{kHz}$ and the maximum amplitude $E_{\max }$ in $\mathrm{mV}$ of the piezoelectric potential against the initial heating-surface temperature $T_{\text {wi }}$. The frequency $f$ and maximum magnitude $E_{\max }$ are determined from Fig. 3. It is seen in Fig. 5 that both $f$ and $E_{\max }$ peak at $T_{\mathrm{wi}}$ of about $300^{\circ} \mathrm{C}$, coinciding with that of most violent miniaturization, as shown in Fig. 3. Timewise, drop minjaturization occurs following large oscillations of the $E$ 


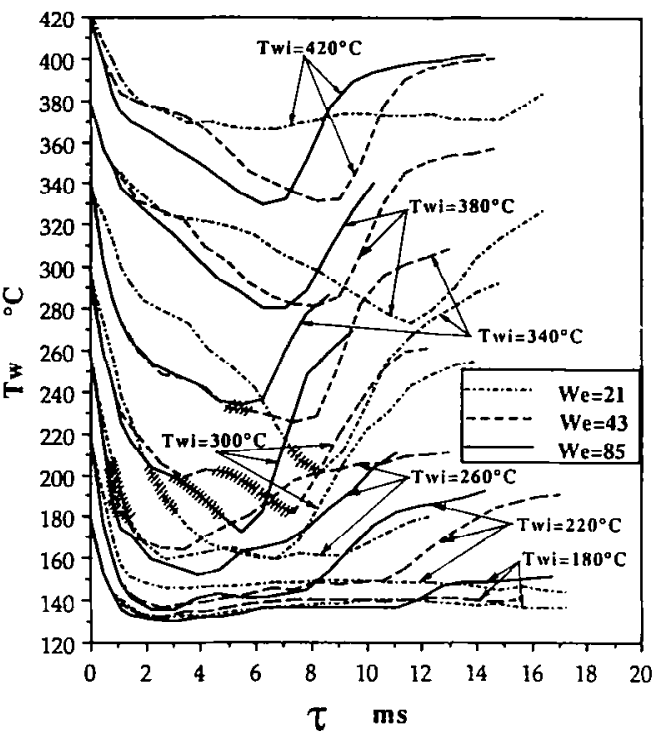

FIG. 4. Temperature-time history of heating surface at different initial temperature and Weber number, slashes indicating occurrence of drop miniaturization.

waves. This implies that the nucleation and growth of vapor bubbles induce the amplification of the $E$ waves, leading to a miniaturization of the drop.

The value of $\beta$ reaches the first maximum at $T_{\mathrm{wi}}$ of about $180^{\circ} \mathrm{C}$. For $T_{w i}$ less or equal to $180^{\circ} \mathrm{C}$, the duration of boiling sounds exceeds the residence time of a drop beyond which a dull sound at a low frequency was generated in the drop. For $T_{w i}$ between 200 and $220^{\circ} \mathrm{C}$, the acoustic pressure is diminished, producing a mild boiling sound. For $T_{w i}$ exceeding $240^{\circ} \mathrm{C}, \beta$ increases, reaches the second maximum value

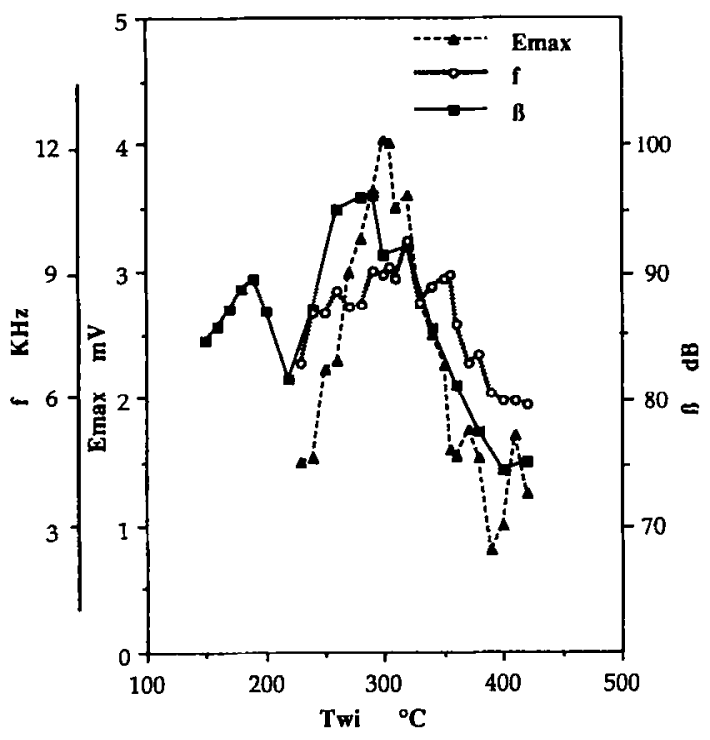

Fig. 5. Maximum values of acoustic pressure $\beta$ and elastic waves (amplitude $E_{\max }$ and frequency $f$ vs $T_{\text {wi }}$. near $T_{\text {wi }}$ of $300^{\circ} \mathrm{C}$, and diminishes with a further increase in $T_{w i}$. The duration of boiling sounds are the same as $E$ wave oscillations seen in Fig. 3 at a given $T_{\text {wi }}$. This means that the generation of boiling sounds is triggered by the liquid-solid contact. Hence, $f$ in Fig. 5 corresponds to the frequency of liquid-solid contacts and may be regarded as the frequency of bubble generation resulting from the liquid-solid contact. It is revealed from Fig. 5 that vapor bubbles are formed at $f$ of $8-9 \mathrm{kHz}$.

\section{Miniaturization mechanism}

Kendall and Rohsenow [17] and Inada and Miyasaka [18] proved analytically that a vapor film between the drop and the heated surface is not stable but always oscillates during the drop residence time as a result of the drop bouncing 3 or 4 times on the vapor film. Hence, liquid-solid contact is not possible unless the vapor film is thin enough. However, no explosive boiling will occur at the stage of the liquid-solid contact even if the contact was realized if the wall temperature was not cooled to a threshold temperature.

Upon a liquid solid contact, once the vapor film is cracked open, a vapor bubble is instantly generated at the threshold temperature as shown in Fig. 6 stage I. At stage II, the bubble grows rapidly. At stage III, the bubble is forced to penetrate through the drop by the inertia force of the liquid surrounding the bubble and departs from the surface. Its stage III triggers the liquid-solid contact spot to move. The departure mechanism of the vapor bubble at stage III can be explained as follows: The liquid surrounds the vapor bubble (states I-II), in which the liquid inertia force acts on the bubble. The drop base is constantly vibrating up and down. The moment following the bottom of the drop departure from the heated surface, the heat supplied from the surface for the growth is severed, the bubble growth stops, and the liquid inertia force acts to force the bubble upward, accelerating the ascending speed of the bubble. For a drop in the state of subcooling, the upward force promoting the bubble ascent is reinforced because the growth velocity of the bubble is diminished owing to condensation.

Figure 7 is a photograph showing the miniaturization of a drop during the explosive boiling on a heated surface at $T_{w i}=320^{\circ} \mathrm{C}$ at $\tau=7 \mathrm{~ms}$. The drop in the liquid film state takes a disk shape. Many minute liquid clusters burst through the liquid film surface like multiple fire crackers.

Henry and Fauske [1] proposed the inertia-force dominating growth of vapor bubbles as the mechanism of boiling fragmentation: as a high-pressure vapor source inside a sessile drop approaches the drop surface opposite the heated surface, the drop explodes, producing a spray of fine liquid clusters. According to the model, the vapor bubble must grow to the size of the drop diameter prior to triggering an explosion. The fragmentation model obviously contradicts the phenomenon seen in Fig. 7. 


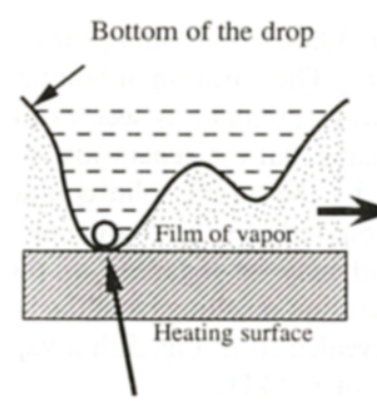

Generation of a vapor bubble I

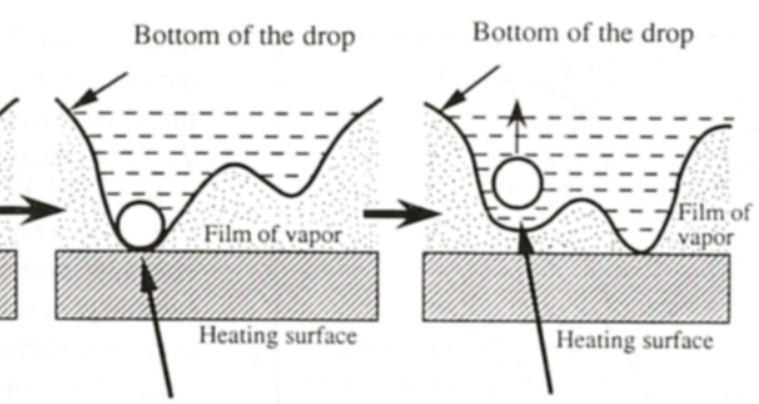

Growth of a vapor bubble II
Emission of a vapor bubble

III

FIG. 6. Model for miniaturization of a sessile drop on a heated surface.

Since the boiling sound is closely related to the generation of minute vapor bubbles which results in miniaturization boiling, the high-frequency boiling sound produced at miniaturization boiling is induced by liquid vibration produced by detaching bubbles.

\section{Heat transfer performance}

An experiment was conducted on a single water drop impinging on a hot polished platinum surface. The experimental setup was described in detail in refs. $[7,11]$ and thus is not repeated here. Figures $8(a)$ and (b) depict heat transfer in the transition boiling regime with the initial drop diameters of 2.3 and $4.0 \mathrm{~mm}$, respectively. Three different subcoolings are superimposed in each figure to illustrate the effects of subcooling on heat transfer performance under the transition boiling state where miniaturization phenomenon occurs. $\bar{T}_{\mathrm{w}}$ and $\vec{q}_{\mathrm{w}}$ denote the time-averaged surface temperature and heat flux, respectively, while $T_{\mathrm{s}}$ signifies the saturated temperature under one atmosphere. The chained line is a nucleated boiling curve for the subcooled pool boiling of water with the two broken lines indicating the transition boiling regime under the subcooling of 30 and $85 \mathrm{~K}$. The solid lines represent the transition boiling regime of drop

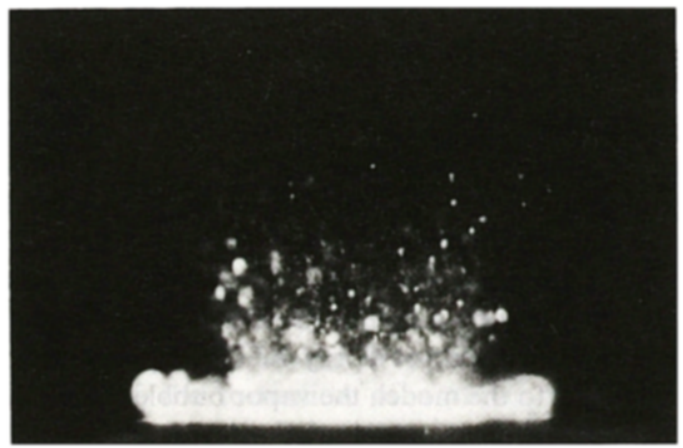

Fig. 7. A photograph of miniaturization phenomena of a drop at $T_{\mathrm{wi}}=320^{\circ} \mathrm{C}$ and $\tau=7 \mathrm{~ms}$. boiling, i.e. the counterpart of the broken lines for pool boiling case. Notice that the nucleate boiling regime of the drop boiling curve is not shown in the figures. It is important to note that the transition regime of drop boiling takes a saddle shape with two peaks (maxima) which is distinctly different from the pool boiling case. One thing in common to both the drop and pool boiling cases is an increase in the heat flux with an increase in the effective superheat $\left(\bar{T}_{w}-T_{\mathrm{s}}\right)$.

\section{CONCLUSIONS}

An experimental study had been conducted on a water drop placed on a heated surface. The initial surface temperature and impingement Weber numbers are varied. It is disclosed that miniaturization occurs in the transition boiling regime. The boiling modes inside the sessile drop are classified into four categories: (i) nucleate boiling commencing at the second half of the drop residence time, which is observed at the maximum boiling-rate point; (ii) latent-heat transport boiling in which many minute vapor bubbles prevail throughout the drop; (iii) miniaturization boiling with many minute liquid clusters bursting through the drop surface; and (iv) thin vapor-film boiling sustained by the vaporization at drop peripheral. The mechanism for the formation of minute vapor bubbles inside a sessile drop observed in the modes (ii) and (iii), resembles that of spontaneous nucleation in pool boiling.

Explosive or miniaturization boiling is observed only in the mode (iii) during which a large number of minute liquid clusters burst through the drop surface. The generation of minute vapor bubbles during the miniaturization boiling induce boiling sounds.

A model relating the intermittent liquid-solid contact and the dispersion of minute vapor bubble, has been proposed to describe the miniaturization mechanism for an explosive boiling in a sessile drop on a heated surface. Its validity is borne out by the experimental observation. 

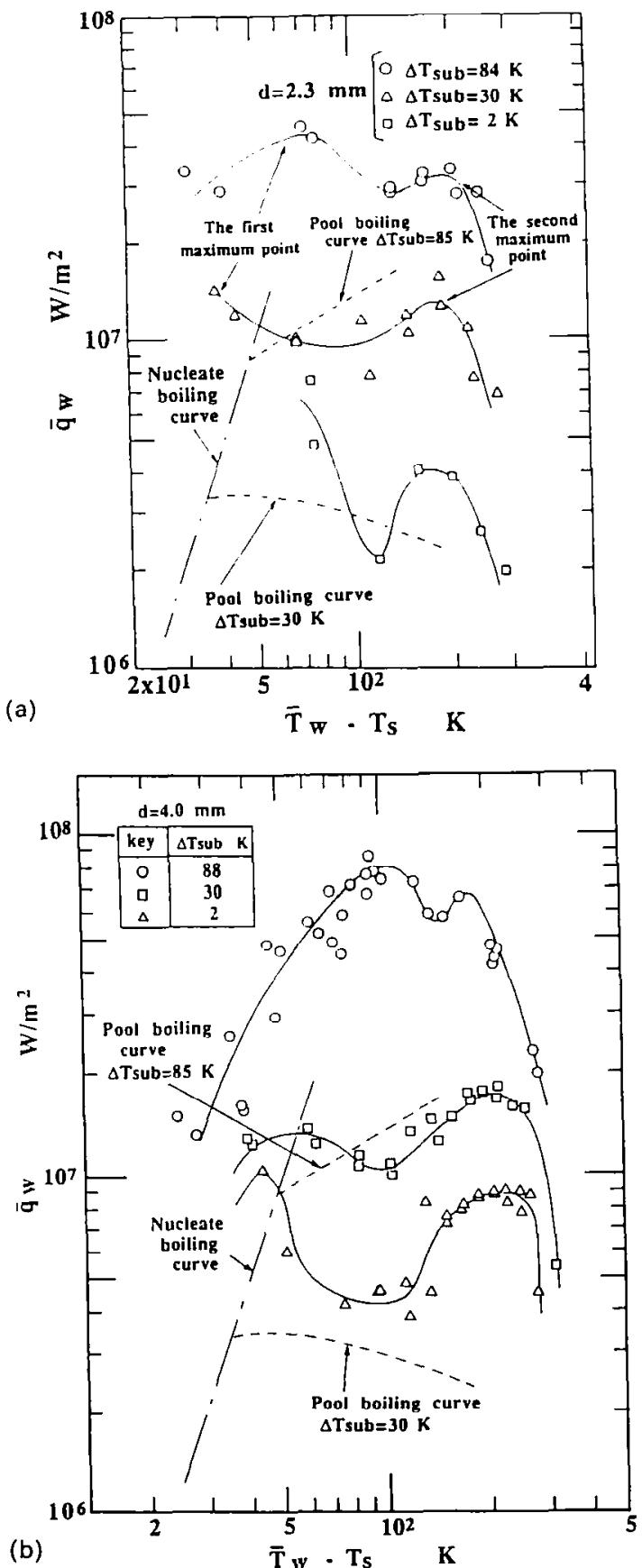

Fig. 8. (a) Drop boiling curves for drop diameters of $d=2.3$ $\mathrm{mm}$. (b) Drop boiling curves for drop diameters of $d=4.0$ $\mathrm{mm}$.

\section{REFERENCES}

I. R. D. Henry and and H. K. Fauske. Nucleation process in large scale vapor explosions, J. Heat Transfer 101, $280-287$ (1979).

2. A. Inoue and S. G. Bankoff, Destabilization of film boiling due to arrival of pressure shock, Part I Experimental, J. Heat Transfer 103,459-464 (1981).

3. A. Inoue. A. Ganguli and S. G. Bankoff. Destabilization of film boiling due to arrival of a pressure shock, Part II Analysis. J. Heat Transfer 103, 465-471 (1981).

4. M. Ochiai and S. G. Bankofi, A local propagation theory for vapor explosion. Paper SNI 6/3, Proceedings of Third Special Meering on Soditum/Fuel Interactions in Fast Reactors, Tokyo (1976).

5. Y. Iida, T. Takashima, T. Watanabe, H. Ohura, C. Ogiso and N. Araki. A fundamental study of thermal interactions of molten salt and cold liquid, Proceedings of 19th National Heat Transfer Symposium of Japan, Nagoya. 5I1-513 (1982).

6. N. Zhang and W.-J. Yang. Evaporation and explosion of liquid drops on a heated surface, Experiments in Fhids 1, 101 III (1983).

7. S. Inada, Y. Miyasaka, K. Nishida and G. R. Chandratilleke. Transient temperature variation of a hot wall due to an impinging water drop. effect of subcooling of the witer drop, Proceedings of ASME/JSME Thermal Engineering Joint Conference (Edited by W.-J. Yang and Y. Mori) 1, $173 \quad 182$ (1983).

8. S. Inada, Y. Miyasaka. K. Sakamoto and K. Hojo, Liquid-solid contact state and fluctuation of the vapor film thickness of a drop impinging on a healed surface, J. Chem. Engng of Jap. 1, 463-468 (1988).

9. S. Inada, K. Nishida, M. Toriba and N. Uchida. Transient heat transfer for water drops impinging on a heated surface (2nd report. Study of liquid-solid contact state based on temperature fluctuation of heated surface). Trans. Jap. Soc'. Mech. Engrs. B 55, 3499-3506 (1989).

10. S. Inada. K. Nishida and T. Kumada, Evaluation of the boiling curve based on mass transfer mechanism for a water drop impinging on a heated surface. In Hear Transfer Science and Techology (Edited by Bu-Xuan Wang). Hemisphere, New York, 287-292 (1988).

11. S. Inada, Y. Miyasaka and K. Nishida, Transient heat transfer for water drops impinging on a heated surface (Ist report, Effects of drop subcooling on the liquidsolid contact state). Bulletin of JSME 28, 2675-2681 (1985).

12. T. Takano and K. Kobayashi, Study of vaporization of a single droplet impinging on a heated ceramic surface, Trans. Jap. Soc. Mech. Engrs. B 53, 1338-1343 (1987).

13. T. Takano and K. Kobayashi, Vaporization behavior of a single droplet impinging on a heated surface with a flame-sprayed ceramic coating, Trans. Jap. Soc. Mech. Engrs. B 54, 1820-2006 (1988).

14. T. Takano and K. Kobayashi, Vaporization behavior of a single droplet impinging on a heated surface with a flame-sprayed ceramic coating-Effect of surface roughness, Trans. Jap. Soc. Mech. Engrs. B 55, 2000-2005 (1989).

15. K. J. Choi and S. C. Yao, Mcchanisms of film boiling heat transfer of normally impacting spray. $I m t$. J. Heat Mass Transfer 30, 311-318 (1987).

16. S. C. Yao, L. E. Hochreiter and K. Y. Cai, Dynamics of droplets impacting on thin heated strips, J. Heat Transfer 110, 214 220 (1988).

17. G. E. Kendall and W. M. Rohsenow. Heat transfer to impacting drops and post critical heat flux dispersed flow, MIT, Tech. Rep. No. 85694-100 (March, 1978).

18. S. Inada and Y. Miyasaka, An analysis of heat transfer based on the drop deformation for nonwetting impinging drop. In Heal Transfer Science and Technology' (Edited by Bu-Xuan Wang). Hemisphere, New York, 661-668 (1987). 